\title{
Una nueva sección en El Debate, tras la Guerra Mundial
}

\author{
Aurora GARCía GonZÁLEZ \\ Universidad de Vigo \\ auroragg@uvigo.es
}

\section{Resumen:}

Seis años después de finalizada la contienda que enfrentó por primera vez a las potencias europeas en un conflicto de envergadura mundial, el diario El Debate, propiedad de la Editorial Católica desde 1912 y bajo la dirección de Ángel Herrera, inició una columna regular que bajo un doble título ("De política inglesa" y "Figuras del Parlamento inglés") comenzó a ofrecer una serie de comentarios sobre cuestiones relativas a la política desde Gran Bretaña. Esta referencia resulta significativa, pese a la falta de perspectiva histórica en aquel momento, ya que manifiesta un cierto giro en las posiciones del periódico. Para ello el diario contrató a un colaborador en Liverpool.

Palabras clave: Periodismo; Guerra Mundia; Política inglesa; El Debate.

\section{A new section in the discussion, after World War}

\begin{abstract}
:
Six years after the end of World War that faced for the first time the European powers on a global scale conflict, the newspaper El Debate, owned by the Catholic Publisher since 1912 and under the direction of Angel Herrera, began a regular column under a double title ("from British politics" and "Figures of the English Parliament") began offering a series of commentaries on political issues from Britain. This reference is significant, despite the lack of historical perspective at the time, as it showed some shift in the stance of the newspaper. This newspaper hired a collaborator in Liverpool.
\end{abstract}

Key Words: Journalism; World War I; British Politics; El Debate.

\section{Referencia normalizada:}

García González, A. (2014): Una nueva sección en El Debate, tras la Guerra Mundial. Historia y Comunicación Social. Vol. 19. Núm. Especial Enero. Págs. 93-106.

Sumario: 1. Introducción. 2. Metodología. 3. El contexto previo y el autor. 4. La nueva columna de opinión. 5. "De política inglesa". 6. "Figuras del parlamento inglés". 7. Conclusiones. 8. Referencias bibliográficas

\section{Introducción}

El objeto de este trabajo es dar a conocer la tarea que se realizó desde aquella columna. El estudio se concentra en el período de un año: desde septiembre de 1923 a septiembre de 1924. La columna, firmada por Nicolás González Ruiz; se publicó por primera vez el 29 de septiembre de 1923 y apareció por última vez el 28 de agosto de 1924. El diario aún publicaría un artículo más referido a la política inglesa en 
septiembre de aquel año, pero ya fechado desde Madrid en vez de Liverpool. Durante el período que se estudia aparecieron 16 artículos dedicados a comentar y difundir información sobre la política en Inglaterra.

En estos artículos se describe el panorama de la política inglesa en aquél concreto momento de su evolución histórica, tras la "extraña" (sic) paz de Versalles. Desde su columna El Debate comentó los conflictos entre liberales, conservadores y laboristas en Inglaterra, las huelgas y los principios socialistas, la crisis industrial y el tratado con Rusia, en suma la problemática que afectaba a la Gran Bretaña en los años inmediatamente posteriores a la gran guerra. En cualquier caso resulta innegable la tarea de difusión llevada a cabo en aquellos momentos, realizada a instancias de un periódico de definidas convicciones, que a lo largo de la contienda había mantenido posiciones claramente conservadoras.

\section{Metodología}

La metodología que se ha seguido para la elaboración de este trabajo ha consistido básicamente en la recopilación de documentación referente al momento, a partir de los ejemplares en papel del diario El Debate correspondientes al año 1923 y 1924, fecha en la que dieron comienzo estas dos secciones.

Tras la localización de los ejemplares, se procedió a su selección y clasificación en dos secciones, o mejor en una sola con un doble título, de los artículos de opinión que permitían ofrecer un punto de vista sobre las posiciones de Gran Bretaña, en esos primeros momentos posteriores a la Guerra Mundial.

Tras una lectura detenida y el correspondiente análisis se ha podido efectuar un repaso a los principales argumentos ofrecidos en su día a los lectores de El Debate. Los resultados se contrataron en un grupo de discusión, dentro de la línea de investigación Teoría e Historia de la Comunicación.

\section{El contexto previo y el autor}

Cuando se inició la Guerra Mundial, la postura oficial de España, con Dato en el poder, fue la neutralidad (ROMÁN, 1997: 269). A pesar de esta neutralidad formal, la clase política y periodística se posicionó abierta y apasionadamente al lado de uno $\mathrm{u}$ otro bando (BARREIRO: 2004).

Los conservadores, principalmente los monárquicos liberales, mauristas y carlistas (integristas y jaimistas), militares y parte del clero se posicionaron del lado "de la autoridad y del orden establecido", virtudes netamente prusianas que a su juicio, defendían los Imperios Centrales. Además no se cansaban de recordar que Inglaterra mantenía en la península el único enclave colonial existente en el continente 
(Gibraltar), que "envenenaba" las relaciones hispano-portuguesas y cómo Francia hacía todo lo posible por recortar la zona española de protectorado marroquí. Es la posición defendida por los diarios El Debate, La Acción, El Universo, Nueva España, El Correo Español, El Día, El Mundo, La Tribuna, El Siglo Futuro, ABC y La Correspondencia Militar. No obstante, y a pesar de esta división genérica, hay que decir que no todos los periódicos se mostraron conformes con la adscripción que se les hace en uno y otro bloque.

En las Cortes de 1916 aparecieron cerca de 40 nombres directamente relacionados con la Prensa, que cubrían un puesto como parlamentarios (GÓMEZ APARICIO: 1974, 447). Todos ellos conocían el papel de la Prensa como configuradora de opinión y utilizaron las páginas brindadas por los diarios, para hacer valer sus puntos de vista ante los lectores. La prensa era un medio fundamental de propaganda y todas las personalidades políticas "contaban e influenciaban" periódicos. No obstante, aunque la Prensa entre 1914 y 1918 abordó fundamentalmente cuestiones de política nacional e internacional, no siempre fueron los periódicos de partido los que difundieron una orientación ideológica concreta; durante la Primera Guerra Mundial en España, algunos partidos tuvieron sus órganos oficiales -caso de El Socialista- pero la mayoría de los periódicos eran empresas "independientes" de las tendencias a las que alentaban.

El diario El Debate, propiedad de la Editorial Católica desde 1912 bajo la dirección de Ángel Herrera, inició una columna regular que con un doble título ofreció una serie de comentarios informativos desde Gran Bretaña. Esa columna estuvo firmada por Nicolás González Ruiz, y se publicó por primera vez el 29 de septiembre de 1923.

El autor que aquí se estudia, Nicolás González Ruiz, era profesor auxiliar de Lengua y Literatura españolas en la Universidad de Liverpool desde 1921. Había empezado en octubre de aquel año como lector de español en la cátedra de Edgar Allison Peers, que desarrollaba una gran actividad en torno a las letras hispánicas. Durante un período de vacaciones de la Universidad, conoció al director de El Debate, Ángel Herrera quien le invitó a escribir para el periódico sobre temas relacionados con su actividad en Liverpool. La colaboración desde Inglaterra no fue larga ya que Herrera, le propondría enseguida la posibilidad de incorporarse a la plantilla de El Debate en España, pero sí fue muy interesante.

En este contexto personal-biográfico se encuadran los artículos que bajo el epígrafe "De política inglesa" tuvieron como objeto proporcionar elementos de juicio a la opinión española sobre las cuestiones más relevantes de la política inglesa en aquellos momentos. En ellos puede muy bien advertirse la huella de la guerra no muy lejana. 


\section{La nueva columna de opinión}

Los textos que se publicaron en esta columna no se limitaron a los asuntos políticos de Inglaterra, lógicamente muy intensamente vividos en aquel momento, sino que recogieron también cuestiones culturales y sociales de aquellos años. Una sociedad, "confrontada a los desafíos de una competencia económica mundial, a una aceleración de los progresos técnicos y a una rebelión que estallaba tanto durante las paralizantes huelgas como en las angustiosas telas de los expresionistas alemanes" (REVAY: 2012).

La columna empezó a abordar asuntos sobre la política inglesa, con ese título como epígrafe, por primera vez el 23 de noviembre de 1923. Los textos que se fueron publicando aparecieron ubicados bajo una doble adscripción "De política inglesa" o bien "Figuras del Parlamento inglés". Los artículos utilizados para este trabajo, que trataban temas políticos y se incluyeron bajo el título "De política inglesa", son 12 según la siguiente relación:

1. El momento actual en la política inglesa $(23 / 11 / 23)$

2. El obscuro porvenir $(02 / 02 / 24)$

3. Momentos difíciles $(23 / 02 / 24)$

4. Lecciones de la realidad (07/03/24)

5. La actuación laborista y la crisis liberal $(03 / 05 / 24)$

6. Nuevo socialismo y viejos partidos (17/05/24)

7. Más sobre la crisis del liberalismo (06/06/24)

8. Antisocialismo (20/06/24)

9. La prueba del socialismo $(17 / 07 / 24)$

10. El tratado con Rusia (17/08/24)

11. La crisis industrial $(28 / 08 / 24)$

12. Divergencias, agitaciones, fieros males (02/09/24)

Otros artículos se destinaron a pergeñar las personalidades de algunas figuras clave del parlamento inglés. Se han empleado en este trabajo los cuatro que se señalan seguidamente:

1. James Ramsey Mac Donald (29/05/24)

2. David Lloyd George (13/06/24)

3. Stanley Baldwin $(29 / 06 / 24)$

4. Winston Churchill (24/07/24)

Estos trabajos describen, con un fin didáctico, el panorama de la situación en Gran Bretaña en los años inmediatamente posteriores al final de la contienda. Del estudio de los artículos publicados en este tiempo es posible obtener un retrato de la situación del país en aquel momento, siempre desde la mirada de un órgano conservador, recién estrenada la Dictadura de Primo de Rivera, en España. 
Es interesante seguir al autor de estos textos porque al hilo de los acontecimientos comentados se presentan con toda nitidez, dentro de las limitaciones de estilo del momento, los intentos del periódico por acercar a los lectores los rasgos generales de la política inglesa y combatir las ideas del partido laborista.

\section{Desde el epígrafe "De política ingles"}

En la etapa que estudia este trabajo, los conservadores tenían una clara mayoría en la Cámara de los Comunes y podían gobernar durante cinco años sin convocar nuevas elecciones, pero Baldwin, como primer ministro, se había sentido obligado a cierta lealtad con su antecesor y optó por convocarlas. Con el país haciendo frente al creciente desempleo, Baldwin convocó elecciones en 1923 en busca de un mandato que le permitiera introducir tarifas proteccionistas para enfrentarse al paro.

\subsection{El momento actual en la política inglesa $(23 / 11 / 23)$}

El primer artículo ya apuntaba lo siguiente: "Las próximas elecciones no van a constituir tan sólo una lucha en la que se decida si van a implantarse o no ciertas tarifas aduaneras; van a ser una lucha por algo mucho más serio aún; por toda una política y una acción social interna y, como consecuencia, por una determinada postura ante el exterior".

Y para contextualizar de cara a los lectores de El Debate se añadía: "Es un poco oscura la cuestión de los partidos políticos ingleses, y, sobre todo, hay, por parte de algunos, el interés equivocado de no ver esa situación tal como es. Hace ahora un año que hubo otras elecciones generales; en ellas, los conservadores obtuvieron la mayoría, no con mucho desahogo; los liberales, divididos, obtuvieron una representación escasa, y los laboristas llevaron al Parlamento una formidable masa de diputados. Esto último valía la pena de llamar la atención, aunque no tanto como un español, no enterado, supondría; los laboristas son gente mansa, y no se proponen devorar ni destruir nada: se contentan con unas reformas trascendentales, suficientes sin embargo, para derribar el maravilloso edificio económico del imperio británico". El texto incluía al final una enseñanza: "Para que saque usted consecuencias aplicables a su país, y estime en lo mucho que vale, la dirección emprendida".

\subsection{El obscuro porvenir $(02 / 02 / 24)$}

Existía el sentimiento común de que lo que ocurría en Inglaterra repercutía en todo el mundo principalmente lo que el diario consideraba "repercusión con frecuencia peligrosísima, porque raras veces son comprendidos desde fuera los diversos momentos por que atraviesa Inglaterra en su evolución".

Así que desde esta columna se aspiraba a esclarecer posibles equívocos: "La primera palabra, el primer concepto, que aparece mal interpretado, a causa del tras- 
plante del suelo inglés al español, es el famoso vocablo moderno: socialismo. Durante estos días he visto en la mayoría de los periódicos ingleses un rótulo que decía: Los socialistas en el Poder. ¿Socialista? En primer lugar, si bien en el Labour Party se alberga nuestro socialismo en Inglaterra, el tono general del partido dista mucho de ser el rojo sangriento, y ahora se ha transformado casi casi en un verdadero color de rosa. Los socialistas ingleses al subir al Poder, lo han hecho de la manera menos socialista posible."

Y como consecuencia el autor suscribía: "Realmente, el Gobierno laborista está en magnífica situación para hacer algo por el prestigio de su país en Europa".

\subsection{Momentos difíciles $(23 / 02 / 24)$}

De ordinario era posible seguir el curso de estos artículos, porque el mismo autor encadena unos con otros: "En un artículo que publiqué en El Debate del día 2 del corriente afirmaba lo peligrosa que sería el arma de la huelga, empleada bajo un Gobierno obrerista. Ahora va a verse esto demostrado de modo terrible - si Dios no lo remedia, y de todo corazón deseamos que lo haga- con la huelga de los obreros de los muelles, que pone a Inglaterra en el trance de quedarse sin los principales artículos de primera necesidad en cuestión de días".

(...) Ésta (huelga) tiene viejos fundamentos: el actual ministro de Transportes, Mr. Harry Gosling, es el ex presidente de la Federación de obreros de Transportes, y como tal dirigió una formidable huelga en 1911. Este es el "hombre del momento", como dicen los diarios ingleses, y quisiéramos saber qué le contestarán sus antiguos compañeros cuando, como miembro del Gobierno, les pida que hagan lo contrario de lo que les pedía que hiciesen cuando era presidente de la Federación.

(...) Al parecer -pues esto no hay quien pueda saberlo a ciencia cierta-, la maniobra de Asquith, el viejo político en todos los sentidos, y de ese formidable y astuto Lloyd George consistía en llevar a Ramsay Mac Donald al Poder para verlo estrellarse.

Como acostumbra, resume en una enseñanza "la cuestión general política, que se cierne por encima de la anterior, consiste en saber si será fácil quitar a los laboristas el Poder, que fácilmente se les dio. Ellos han de emprender, tarde o temprano, el camino de las reformas trascendentales, y aunque rebajen mucho despertarán la enemiga de la mayor parte del país."

\subsection{Lecciones de la realidad (07/03/24)}

A lo largo de todo el año no cejó en su objetivo: "Ocupémonos una vez más de este gobierno Socialista, que está conquistando a la opinión inglesa precisamente por ser mucho menos socialista de lo que se esperaba.

Los comentarios se apoyaban con frecuencia en los periódicos ingleses: "Copiemos un interesante párrafo escrito por el famoso redactor político de The Observer, J. L. Garvín. 
"Si los laboristas acudiesen ahora en consulta al país, con un programa no partidista y sí solamente de administración y trabajo, obtendrían la confianza de la mayoría; pero si hablasen de nacionalización, o leva del capital, o cualquier otro punto fundamental del programa que enarbolaron en las últimas elecciones, el partido laborista sufriría la más violenta caída y no podría reponerse en muchos años." Esta es la opinión de todos, según he podido observar.

Y en como siempre para concluir: "La lección que la realidad nos ofrece en este pueblo verdaderamente democrático es elocuentísima, y ojalá fuese aprovechada por los españoles. Las huelgas y otras perturbaciones sociales hablarán por él (laborismo) y demostrarán su esterilidad".

\subsection{La actuación laborista y la crisis liberal (03/05/24)}

"Míster Ramsay Mac Donald ha dado a entender en un reciente discurso que el momento de las próximas elecciones inglesas pudiera tardar todavía dos o tres años en llegar. Esta insinuación ha levantado horribles y furiosas tempestades en todos los sectores políticos.

Todo el ambiente político inglés se hallaba conmovido ante la idea de que pudiera permanecerse mucho tiempo en el estado transitorio e inestable que supone el no haber en el Parlamento ninguna mayoría.

"Entre las plagas más amenazantes y más inmediatas que se ciernen sobre el liberalismo inglés se halla un furor unionista, que ha entrado a muchos de sus miembros. Este unionismo se manifiesta, por una parte, en una inclinación al laborismo que origina la deserción de muchos calificados liberales que van a engrosar las huestes de Ramsay Mac Donald y, por otra, en un recrudecimiento, de aquellas viejas amistades que juntaron a liberales y conservadores para formar el gran bloque gubernamental.

Los conservadores callan y esperan, y se sonríen un poco de los apuros liberales, mientras esperan el instante decisivo del gran choque contra el laborismo, que es el enemigo a combatir. Por su parte, el laborismo trata de apoderarse de las armas conservadoras y herir al conservadurismo con ellas. Es buena idea, y seguramente a estas horas ha producido, por lo menos, el gran resultado de que todo el mundo pierda el miedo al fantasma socialista".

\subsection{Nuevo socialismo y viejos partidos $(17 / 05 / 24)$}

La referencia constante al socialismo aparece en contraste con los partidos españoles de larga tradición: "Nos hallamos ante un socialismo nuevo y en el fondo - he ahí el peligro oculto- quizá no menos socialista que el que más; pero este socialismo lo dirigen hombres cultos y serenos que comprenden que el primer servicio que debe hacerse al pueblo y el más apreciado de todos es mantenerlo dentro de la paz y del orden." Para describir la situación alude a Baldwin y Asquith. "Cada uno de estos jefes políticos se ha dirigido a su partido en magnífico y brillante discurso; pero ambos han demostrado una cosa que hace algún tiempo venía insinuándose y que 
ahora se hace patente: los viejos partidos gubernamentales ingleses están gastados, y su actual orientación los conduce a un desgaste progresivo que amenaza terminar para los liberales - como ya se dijo- en una completa anulación".

5.7. Más sobre la crisis del liberalismo (06/06/24)

Se habla de elecciones en plazo no lejano en todos los periódicos ingleses: los laboristas hablan con calma, los conservadores con serenidad, los liberales angustiosamente. "Dos elecciones parciales, una en Glasgow y otra en Liverpool, han tenido lugar recientemente, y su resultado ha de servir de lección provechosa se quieren y saben aprovecharla los que deben y pueden.

Consecuencias: a) la unión de las gentes de orden en un bloque antilaborista triunfará siempre, aun en los distritos de un socialismo más rabioso. b) en toda lucha triangular los liberales obtendrán una votación ridícula, que demostrará la polarización de las fuerzas nacionales hacia dos grandes núcleos y la inutilidad de los enjuagues, medias tintas y paños calientes".

Estas consecuencias aparecen tan claras y se imponen a todos de tal manera, que la política del Gobierno no se dirige a otra cosa que a evitar diplomáticamente la unión de conservadores y liberales, y absorber entretanto la sangre del partido liberal, a cuya costa engorda y echa pelo el laborismo de una manera portentosa. Asquith y Lloyd George ven esto perfectamente, y toda su actividad se orienta hacia la petición de elecciones.

Entretanto se dice que las elecciones serán en otoño. Lo dicen todos menos el Gobierno, que necesita primero llevar a cabo su tarea de anulación y absorción del partido liberal".

\subsection{Antisocialismo (20/06/24)}

Los partidos políticos actuales son incapaces de contrarrestardebidamenteel esfuerzo realizadoporloslaboristas.(...)Estolohanvistodoshombres,omejordicho, lohavistounoChurchill- inmediatamente, $\mathrm{y}$ ha tratado de hacer de ello el pedestal que le clave y le saque de su actual situación. El otro -Lloyd George- vio las cosas también desde un principio; pero cometió el error de considerar que el partido liberal podía ser la panacea curativa, y congregar bajo su bandera a todos os contrarios de socialismo. Ahora está convencido de su equivocación, y trata de ganar a Churchill por la mano; pero es lo cierto que ambos adelantan poco: la opinión les considera quizá como los dos políticos más políticos (en aquel sentido que los españoles no olvidaremos nunca por el daño que nos ha hecho), y desconfía de ellos, no viendo nada más que maniobras interesadas tras sus movimientos.

Las elecciones se avecinan y un laborismo actuando desde el Poder con Ramsay Mac Donald como jefe y luchando contra organizaciones viejas, desacreditadas y desacordes, puede muy bien obtener una mayoría que atraiga sobre Inglaterra días inciertos y obscuros, que serán mucho más terribles, porque el buen inglés no está 
acostumbrado a ellos y casi no cree en su existencia. Este hecho es elocuentísimo para todos los que quieran entender; el laborismo, conforme adquiere fuerza, va arrojando la máscara y manifestándose en realidad más de acuerdo con su programa, o, por lo menos, con el programa predicado en la oposición. Su actuación ante la mencionada huelga ha sido tan clara, que hasta periódicos siempre benévolos con el laborismo se han creído en el caso de indignarse.

\subsection{La prueba del socialismo $(17 / 07 / 24)$}

El socialismo inglés continúa dando su lección al de toda Europa, y al de España particularmente. Ha demostrado que siente el patriotismo, y que, aun estando dispuesto a realizar lo posible de su programa, lo pierde de vista en cuanto entran en juego altos intereses nacionales, infinitamente superiores a todos los partidos. Y es una buena y sana lección, y una prueba que no debe olvidarse, sino, al contrario, publicarse repetidamente y a los cuatro vientos: en cuanto un socialista quiere servir a su país y trabajar por la grandeza de su Patria, lo primero que tiene que abandonar es el programa socialista.

\subsection{El tratado con Rusia (17/08/24)}

Lo que aparece claro, por lo pronto, es que la opinión está fundamentalmente dividida en dos grandes sectores. Uno de ellos, más o menos disimuladamente afecto al laborismo y del que pudiéramos considerar intérprete al gran periódico The Obser$v e r$, se esfuerza en demostrar que el convenio es un acierto absoluto que la nación debe agradecer vivamente a Ramsay Mac Donald.

El grupo financiero, el partido conservador y periódicos anfibios y acomodaticios, pero de gran difusión, como el Daily Mail, arremeten contra el Ramsay Mac Donald y señalan los puntos flacos del convenio, sus grandes errores y hasta ven en él peligros y amenazas, no por cierto sin razón para ello, como se va a ver.

El grupo de financieros encuentra disparatado que Inglaterra acuda con su crédito y su garantía a proporcionar a Rusia un empréstito que pudiera elevarse hasta 500 millones de libras. Esto, tratándose de un país como Rusia, actualmente de una insolvencia absoluta, gobernado por quienes no estiman como esenciales ni la buena fe al hacer los contratos, ni la honradez al cumplirlos, es ciertamente un peligro muy grave.

\subsection{La crisis industrial $(28 / 08 / 24)$}

La creciente gravedad de la crisis industrial inglesa, y sobre todo la forma como contribuyen a ella los obreros, atentos sólo a tener mejoras de sueldo, sin ver como esa incesante subida se va transformando en un encarecimiento general de todo lo que se necesita para vivir, que determina una más angustiosa situación a la vuelta de poco tiempo. 
Tomamos las cifras de una interesante estadística que publicaba el Evening Standart.

- Electricistas.- Irán muy en breve a la huelga, si se rechazan, como es probable, sus peticiones. Desean diez chelines de aumento a la semana. El paro afectará a 300.000 hombres.

- Maquinistas.- Reclaman una libra esterlina de aumento a la semana. Han obtenido aumentos recientes, y parece difícil que obtengan el que piden ahora. La huelga envolverá a 500.000 hombres.

- Empleados de las estaciones de ferrocarril.- Piden diez chelines semanales de aumento. El próximo viernes se resumirá la comisión encargada de buscar arreglo. Si no lo hay, lo cual no sería difícil, irán a la huelga al fin de esta semana 200.000 hombres.

- Ramo de construcción.- Están en huelga hace algún tiempo la may9or parte de lo obreros de esta ramo. Se anuncia que ahora van a unírseles los que aún estaban trabajando. Entre los que huelgan y los que holgarán son 350.000 hombres.

Londres está amenazado, además, de una serie de conflictos particulares. Los conductores de taxis piden ahora diez chelines más por semana. Si van a la huelga pararán 50.000 hombres y quedará una ciudad como Londres sin un servicio de tan gran importancia.

\subsection{Divergencias, agitaciones, fieros males $(02 / 09 / 24)$}

El último esfuerzo por reunir datos sobre la situación inglesa, consciente de tratarse sólo de un esbozo, resumía. "Las gentes se encuentran ante unos partidos fracasados que no comprenden la necesidad de renovarse y un movimiento turbio e indeciso, si se quiere, pero lo bastante atrevido como para salir enarbolando bandera de remedio para todos los males. Las soluciones laboristas, aplicadas íntegramente, representarán la destrucción de toda economía inglesa".

\section{6. "Figuras del parmalento Inglés"}

Otros cuatro artículos ofrecieron a los lectores de El Debate los retratos a grandes trazos de los principales representantes de las fuerzas políticas en liza: conservadores, liberales y laboristas a las que se añadió por razones obvias y de proximidad temporal la de Winston Churchill. En trazos rápidos y, en ocasiones, con buen humor se destacaron las principales notas descriptivas de los cuatro representantes con un artículo dedicado a cada uno de ellos: 


\subsection{James Ramsey Mac Donald}

Era un político de origen humilde, que había sido el líder del Partido Laborista en la Cámara de los Comunes en 1914. Su oposición a la I Guerra Mundial le había hecho impopular. Regresó al Parlamento en 1922, cuando los laboristas superaron a los liberales como el partido más grande del ala izquierda del espectro político en el Reino Unido. Su primer Gobierno -formado con el apoyo de los liberales en 1924duró nueve meses. Se trataba de un hombre imprevisible al que en esta columna se describía así:

La formidable incógnita de lo que es el buen Mac encierra quizá el porvenir de Inglaterra. (...) Por eso sobre el porvenir de Inglaterra, se cierne una interrogación. Churchill, el hombre que más bravamente ha empuñado la bandera antilaborista, se pregunta en un artículo que publica The Weekly Dispatch de hoy, refiriéndose a Ramsay Mac Donald: ¿Qué hará? Esta pregunta contiene el secreto del futuro. (El Debate, 20/05/24)

\subsection{David Lloyd George}

Antes de las nuevas elecciones de 1923, este hábil político había zanjado viejas rencillas con Asquith, lo que había servido para reunificar el partido liberal. Esto le permitió presentar un frente unido contra el proteccionista Stanley Baldwin. Sin embargo, en las elecciones de 1924, aunque fue reelegido, los liberales fueron barridos y quedaron como un minúsculo grupo en la Cámara de los Comunes, del que fue elegido portavoz. González Ruiz rompe una lanza en su defensa y lo estudia como parlamentario y como periodista del que puede decir:

El de las conferencias internacionales es temible; díganlo Briand y Poincaré, (...) La aventura francesa - según Lloyd George- alejaba indefinidamente la posibilidad de cobrar las reparaciones. Hoy, que se conoce el informe de los peritos, y que los lectores de El Debate están instruidos en esta cuestión por los luminosos artículos de don Francisco Cambó, me parece oportuno recordar esta otra ocasión en que Lloyd George tuvo razón contra la mayoría, mientras el Gobierno de Baldwin con sus lenidades contribuía a la disminución del prestigio político de Inglaterra.

Sin embargo, el específico liberal que este gran hombre preconiza tiene tan escasa eficacia que son muy pocos los que creen en él. (El Debate, 13/06/24)

\subsection{Stanley Baldwin}

A finales de 1922 se había roto la coalición entre conservadores y el partido liberal Lloyd George. Se nombró a Baldwin Chancellor of the Exchequer (Ministro de hacienda británico). En las elecciones de noviembre, los conservadores ganaron por mayoría.

Baldwin era solamente lo que aquí llaman "un gran conservador”, expresión felicísima y que se aplica indistintamente a los hombres de todos los partidos cuando sintetizan y personifican de manera excelente las cualidades fundamentales de la agrupación a que pertenecen. (...) La estela de desaciertos que, según muchos, 
siguió inseparablemente a míster Baldwin- rira bien qui rira le dernier-, no se ha debido más que a su extraordinaria sinceridad y carencia de la más elemental "mano izquierda" política. (El Debate, 29/06/24)

\subsection{Winston Churchill}

En 1923 Churchill se había vuelto a presentar como candidato liberal en el distrito de Leicester, perdiendo nuevamente. Más tarde se fue acercando al Partido Conservador, si bien se autonombró «antisocialista» $\mathrm{y}$ «constitucionalista».

El autor justifica así la referencia a Churchill: "Perdida la elección en Westminster por una minoría insignificante, Churchill no puede ser considerado parlamentario, ateniéndonos a la letra; pero considerando la importancia política del famoso ex ministro y teniendo en cuenta que no deja de influir activamente en la vida pública con su actuación constante, no parecerá demasiado el hacerle figurar aquí." Tras señalar que suele reconocerse a Churchill con el calificativo clever añade:

Siempre hay que calificarle con una palabra empezada por ex: Churchill el ex ministro, el ex liberal, el ex conservador..., el extraviado, añadiría yo, si se me permitiese el chiste. (...) En los partidos actuales no cabe: no podría ser jefe de ellos, ni quiere ser uno más entre los innumerables cabecillas de ellos. Por eso anda buscando postura, y buscándola ha encontrado la mejor que no podía escaparse a su claro talento: ha comprendido que un partido nacional antisocialista lograría reunir entorno un núcleo formidable de opinión. (El Debate, 24/07/24)

\section{Conclusiones}

Del recorrido por este breve periodo de tiempo se pueden inferir las siguientes conclusiones:

- Resulta innegable la tarea de difusión llevada a cabo en aquellos momentos realizada a instancias de un periódico de definidas convicciones, que a lo largo de la contienda se situaba en posiciones claramente conservadoras.

- Esta tarea de materializó en El Debate a través de la sección que firmaba Nicolás González Ruiz que apareció el 29 de septiembre de 1923 por primera vez y se publicó regularmente con el doble título "De política inglesa" y "Figuras del parlamento inglés".

- Desde esta columna de El Debate se abordaron los conflictos entre liberales, conservadores y laboristas en Inglaterra, las huelgas y los principios socialistas, la crisis industrial y el tratado con Rusia, en suma la problemática que afectaba a la Gran Bretaña en los años inmediatamente posteriores a la gran guerra. 
- Esta tarea estuvo marcada en todo momento por una declarada aversión al partido laborista.

\section{Bibliografía}

\subsection{Libros}

BARREIRO, C. (2004) "La prensa Española ante la primera guerra mundial" en revista Arbil No 78. http://www.arbil.org/(78)cris.htm [24/04/2013].

GARCÍA GONZÁLEZ, A. (1997) Nicolás González Ruiz, periodista y escritor. Vimón, Pontevedra,

GÓMEZ APARICIO, P. (1974) Historia del Periodismo Español. Editora Nacional, Tomo 3. Madrid.

GONZÁLEZ RUIZ, N. (1943) "Autocrítica" en Madrid, 6-V

IGLESIAS, F. (1980) Historia de una empresa periodística, Prensa Española. Prensa Española. Madrid.

MARAÑÓN, G. (1957) Prólogo a la Obra Selecta de Nicolás González Ruiz. Ed. Labor. Barcelona.

REVAY, TH. (2012) El último verano en Mayfair. Vergara. Barcelona.

ROMÁN, M. (1997) Historia De la empresa de La Voz de Galicia (1889-1939) Servicio de Publicaciones de la Universidad de Vigo. Vigo.

ROMÁN, M. (1999), "Periodistas y políticos. El Congreso de los Diputados en 1909 y en 1996" en BARRERA, C. Del gacetero al profesional del periodismo. Fragua. Madrid.

SÁNCHEZ ARANDA, J.J. y BARRERA DEL BARRIO, C. (1992) Historia del Periodismo español. Eunsa. Pamplona.

SEOANE, M.C y SÁIZ, M.D. (1996) Historia del Periodismo en España. T. 3: El siglo XX: 1898-1936. Alianza Editorial. Madrid.

8.2 Diarios

El Debate 1923-1924

\section{La autora}

Aurora García González es doctora en Comunicación Pública. Como profesora titular de Periodismo imparte la materia "Opinión Pública", en el Grado de Publicidad y Relaciones Públicas, en la Facultad de Ciencias Sociales y de la Comunicación de la Universidad de Vigo.

Ha publicado diversos trabajos de investigación en el campo de la comunicación, entre otros: "Radio digital e interactiva. Formatos y prácticas sociales" en Icono 
14 Vol. $\mathrm{n}^{\circ} 15$ Marzo 2010 (ISSN: 1697-8293); "Les programmes de radio au point du jour, un service public. Analyse de quatre programmes de la participation des émetteurs en Espagne" en Recherches en communication Vol. $\mathrm{n}^{\circ} 26$ Noviembre 2008 (ISSN: 1370-0480) o "Comunicación institucional pública en la radio: Galicia, elecciones 2004” en Sphera Pública Vol. nº 52005 (ISSN: 1180-9210). 